\title{
Assessment of submicroscopic infections and gametocyte carriage of Plasmodium falciparum during peak malaria transmission season in a community-based cross-sectional survey in western Kenya, 2012
}

Zhiyong Zhou ${ }^{1 *}$, Rebecca M. Mitchell ${ }^{1}$, Simon Kariuki², Christopher Odero², Peter Otieno ${ }^{2}$, Kephas Otieno $^{2}$, Philip Onyona ${ }^{2}$, Vincent Were ${ }^{2}$, Ryan E. Wiegand ${ }^{1}$, John E. Gimnig ${ }^{1}$, Edward D. Walker ${ }^{3}$, Meghna Desai ${ }^{1}$ and Ya Ping Shi ${ }^{1 *}$

\begin{abstract}
Background: Although malaria control intervention has greatly decreased malaria morbidity and mortality in many African countries, further decline in parasite prevalence has stagnated in western Kenya. In order to assess if malaria transmission reservoir is associated with this stagnation, submicroscopic infection and gametocyte carriage was estimated. Risk factors and associations between malaria control interventions and gametocyte carriage were further investigated in this study.
\end{abstract}

Methods: A total of 996 dried blood spot samples were used from two strata, all smear-positives (516 samples) and randomly selected smear-negatives (480 samples), from a community cross-sectional survey conducted at peak transmission season in 2012 in Siaya County, western Kenya. Plasmodium falciparum parasite presence and density were determined by stained blood smear and by $18 \mathrm{~S}$ mRNA transcripts using nucleic acid sequence-based amplification assay (NASBA), gametocyte presence and density were determined by blood smear and by Pfs 25 mRNA-NASBA, and gametocyte diversity by Pfg377 mRNA RT-PCR and RT-qPCR.

Results: Of the randomly selected smear-negative samples, $69.6 \%$ (334/480) were positive by 18S-NASBA while 18S-NASBA detected $99.6 \%$ (514/516) smear positive samples. Overall, $80.2 \%$ of the weighted population was parasite positive by 18 S-NASBA vs $30.6 \%$ by smear diagnosis and $44.0 \%$ of the weighted population was gametocyte positive by Pfs $25-$ NASBA vs $2.6 \%$ by smear diagnosis. Children $5-15$ years old were more likely to be parasitaemic and gametocytaemic by NASBA than individuals $>15$ years old or children $<5$ years old while gametocyte density decreased with age. Anaemia and self-reported fever within the past $24 \mathrm{~h}$ were associated with increased odds of gametocytaemia. Fever was also positively associated with parasite density, but not with gametocyte density. Antimalarial use within the past 2 weeks decreased the odds of gametocytaemia, but not the odds of parasitaemia. In contrast, recent anti-malarial use was associated with lowered parasite density, but not the gametocyte density. Use of ITNs was associated with lower odds for parasitaemia in part of the study area with a longer history of ITN interventions. In the same part of study area, the odds of having multiple gametocyte alleles were also lower in individuals using ITNs than in those not using ITNs and parasite density was positively associated with gametocyte diversity.

\footnotetext{
${ }^{*}$ Correspondence: zaz6@cdc.gov; yps0@cdc.gov

${ }^{1}$ Malaria Branch, Division of Parasitic Diseases and Malaria, Center

for Global Health, Centers for Disease Control and Prevention, Atlanta,

GA, USA

Full list of author information is available at the end of the article
} 
Conclusion: A large proportion of submicroscopic parasites and gametocytes in western Kenya might contribute to the stagnation in malaria prevalence, suggesting that additional interventions targeting the infectious reservoir are needed. As school aged children and persons with anaemia and fever were major sources for gametocyte reservoir, these groups should be targeted for intervention and prevention to reduce malaria transmission. Anti-malarial use was associated with lower parasite density and odds of gametocytaemia, but not the gametocyte density, indicating a limitation of anti-malarial impact on the transmission reservoir. ITN use had a protective role against parasitaemia and gametocyte diversity in western Kenya.

Keywords: Plasmodium falciparum, Gametocytes, Risk factors, Antimalarials, ITNs, Kenya

\section{Background}

Scale-up of malaria control interventions in endemic countries has resulted in drastic declines in malaria morbidity and all-cause mortality in many African countries. In western Kenya, after decades of malaria prevention and treatment measures, such as insecticide-treated nets (ITNs), intermittent preventive treatment in pregnancy (IPTp) and artemisinin-based combination therapy (ACT), the prevalence of Plasmodium falciparum in children below 5 years of age as diagnosed by blood smears declined from $60 \%$ in 2003 to $26 \%$ in 2008, but rose to $41 \%$ in 2009 [1]. Malaria prevalence was $38 \%$ in children $<5$ years in 2010 [2]. It is unclear if this reversal and stagnation in malaria prevalence in this area is associated with submicroscopic infections and the sexual stage reservoir, gametocytes.

Malaria transmission relies on sexual stage parasites, the gametocytes that derive from a small fraction of asexual parasites. Immature forms of $P$. falciparum gametocytes (stage I-IV) are sequestered in organ tissue, mainly in the bone marrow [3]. The gametocyte maturation process in the bone marrow requires $10-12$ days [3, $4]$, and mature gametocytes then release into peripheral blood and persist with an average circulation time of 4.66.5 days [5-7] using microscopy. An average duration of gametocytaemia has been reported at 55 days (95\% CI 28.7-107.7) using molecular methods following nonACT drug treatment [6]. The mature gametocytes (stage V) which are responsible for parasite transmission from human to mosquitoes often circulate at low densities [8]. In this context, sensitive molecular detection tools could improve detection of low densities of gametocytes and submicroscopic infections to identify potential transmission reservoir. RNA-based detection methods, such as real-time quantitative nucleic acid sequence based amplification (NASBA) technology, are widely used and highly sensitive with a quantitative detection limit of about 20 parasites/ml blood for research purposes [9]. The Pfs25NASBA can detect 3- to 10-fold more gametocytes than microscopy [10]. It has been shown by the highly sensitive molecular methods that the gametocyte reservoir is much larger than previously detected or reported $[8,10]$.
Gametocyte production and epidemiology could be associated or influenced by several factors that include transmission intensity, exposure to interventions like ITNs and anti-malarial drugs, host age/immunity, asexual parasite density, anaemia, multiplicity of infection [4, 11-15]. In high malaria transmission areas, gametocyte carriage is most prevalent in children under 5 years of age [4] and declines with increasing age in parallel with asexual parasite prevalence and densities due to increased host anti-parasite immunity $[16,17]$. In areas of low transmission intensity, gametocyte prevalence is low among all age groups [18] and the density of gametocytes relative to that of asexual parasites increases with age $[4,19]$. Studies have reported a positive association between gametocyte density and the proportion of infected mosquitoes [20]. However, some reports have shown that mosquito infection is not directly proportional to the density of gametocytes in human blood and submicroscopic gametocytes could also infect mosquitoes and sustain malaria transmission $[4,10,20-22]$.

Anti-malarials are also found to affect malaria transmission $[4,15,23,24]$. Artemisinin derivatives are very effective in clearing asexual parasites and reduce immature gametocytes, but may not affect mature gametocytes $[25,26]$. Artemether-lumefantrine (AL), was officially introduced in Kenya in 2004 as the first-line drug for treatment of uncomplicated malaria but extensively implemented in 2006 including western Kenya [27]. In western Kenya, a large proportion of asymptomatic infections are associated with submicroscopic parasite densities [28]. A cohort study conducted in rural Kisumu County in western Kenya has reported that treatment of asymptomatic parasitaemic individuals with AL reduced the proportion of individuals who became gametocytaemic during the first 12 weeks of follow-up [29]. However, other studies showed that residual submicroscopic gametocytes after treatment with ACT occurred commonly in Kenya and was associated with a longer duration of gametocyte carriage and a higher transmission potential $[7,28]$.

ITNs have been deployed for over 16 years and remain the primary malaria intervention in western Kenya. ITNs 
significantly reduce malaria morbidity and all-cause mortality in children less than 5 years old [30,31]. ITNs also suppress the mosquito populations and reduce their ability to transmit malaria by $70-90 \%$ [32]. An earlier study has shown that multi-clone P. falciparum gametocytes can persist three times longer than those from single-clone infections [33]. Multiple clone infections may increase the male clones in female-biased sex ratio of gametocytes [4, 34 and clones with a male-biased ratio were more infectious to mosquitoes in vitro $[34,35]$. However, although ITNs have proved to be an efficacious and cost-effective vector control intervention to reduce clinical disease and malaria transmission, their long term impact on gametocyte carriage and gametocyte diversity is unknown.

Given the complex biology and epidemiology of gametocytes that could be influenced by multiple factors including ITN use and anti-malarial treatment, the objectives of this study were: (1) to estimate the level of submicroscopic infection and gametocyte carriage in circulating blood, measured by molecular tools, in a region of western Kenya, where malaria transmission has stagnated since 2009, (2) to assess the risk factors associated with gametocytaemia, and (3) to determine the associations between malaria control interventions and gametocyte carriage. Results from this study could be useful in understanding and improving the interventions on transmission reservoirs and in providing the information for development of new strategies for transmission reduction and elimination of malaria.

\section{Methods}

\section{Study area and population}

This study was part of a community-based annual cross sectional survey to evaluate impact of malaria control interventions on malaria parasitaemia and anaemia within the KEMRI/CDC Health and Demographic Surveillance System (HDSS) [36]. The cross sectional survey was conducted during peak malaria transmission season from June to July of 2012 in the two adjacent areas of Asembo (Rarieda district) and Karemo (Siaya district), Nyanza region of western Kenya. Use of ITNs has been consistently high in the Asembo area since 1997 while ITNs were introduced in Karemo in 2004 and scaled up in 2006. The entomological inoculation rate (EIR) estimated by pyrethrum spray catches has been consistently at $<10$ infectious bites per person per year since 2008 (CDC, unpublished data) although recent estimates of EIR through human landing catch indicate the pyrethrum spray catches may underestimate EIRs [37].

\section{Survey data collection and sample selection}

The sampling frame for year 2012 survey included all households in the study areas with at least one child under 5 years old. From this sampling frame, households were selected randomly via probability sampling and all individuals above 1 month of age were sampled in each selected household. A total of 1779 samples was collected for this survey. Self-reported information on age, fever, ITN and recent anti-malarial use were collected based on the study questionnaire. Participants were categorized into three age groups ( $<5$ years old, $5-15$ years old, and $>15$ years old). Fever was defined as self-reported fever within the past $24 \mathrm{~h}$. ITN use was defined as having slept under an ITN the night prior to the survey. Recent anti-malarial (AM) use was defined as receiving antimalarials (96\% had taken AL) in any dosage at any time within the 2 weeks prior to the survey. During the survey, finger prick blood sample was collected to prepare blood smears to determine the presence of parasitaemia and gametocytaemia by microscopy [38]. Haemoglobin level was determined using portable HemoCue photometers (HemoCue AB, Angelholm, Sweden).

In addition, $50 \mu \mathrm{L}$ of whole blood was collected into each spot of a Whatman 903 filter paper and dried overnight at room temperature. The dried blood spot (DBS) was sealed tightly with desiccants and a moisture indicator, and shipped to the CDC laboratory in Atlanta, USA within 2 months after sample collection and stored at $-80^{\circ} \mathrm{C}$ until use.

For the present study, a total of 999 DBS samples from 1779 samples collected during the survey were tested to determine submicroscopic infection and gametocyte carriage. Among them, 446 samples were from Asembo, including all 221 smear-positive and a random selection of 225 from 600 smear-negative samples, and 553 samples were from Karemo, including 298 smear-positive and 255 from 660 randomly selected smear-negative samples. Random sampling of the smear negative samples was performed by Random Sample Excel Professional plus 2010 in Excel, based on the sample size calculated by the program online [39].

\section{Laboratory tests \\ Nucleic acid extraction}

Total RNA was extracted from DBS samples using QIAshredder and RNeasy mini kits (QIAGEN, Valencia, CA) according to the QIAGEN protocols. A whole spot from a DBS sample was cut and used for RNA extraction. After RNA extraction, DNase digestion on one of three aliquots of RNA sample was performed with Ambion DNA-free DNA removal kits (Thermo Fisher Scientific, Waltham, MA) for use in Pfg377 reverse transcription polymerase chain reaction (RT-PCR) and RT-qPCR assays. The other two RNA aliquots without Ambion DNA-free kit treatment were used for 18S-NASBA and Pfs25-NASBA assays. 


\section{Total parasite load detected by 18S-NASBA}

The mRNA transcripts of $18 \mathrm{~S}$ small subunit rRNA gene of $P$. falciparum were measured for detection of malaria infections and submicroscopic parasites. The primers and molecular beacon probes of 18S-NASBA used were based on previously published methods [9]. The assay was performed on EasyQ analyser (BioMerieux, Durham, NC) using the Nuclisens Basic Kit in a total reaction volume of $10 \mu \mathrm{L}$ per reaction at $41{ }^{\circ} \mathrm{C}$ for $90 \mathrm{~min}$. Positivity was calculated with the time-to-positivity based on the time-point of amplification at which the fluorescence passed a given threshold (above the mean fluorescence of three negative controls plus 20SD) as described by Schneider et al. [9]. In order to quantify the parasite density in samples, a standard curve was made in duplicate by 10 -fold dilution series $\left(10^{4}-10^{-2}\right.$ parasites $/ \mu \mathrm{L}$ of blood) from cultured 3D7 ring stage parasites. The limit of quantification (LOQ) for 18S-NASBA was 0.01 parasites $/ \mu \mathrm{L}$ blood based on the standard curve established in this laboratory.

\section{Mature stage V gametocyte detected by Pfs25-NASBA}

Pfs25 mRNA was used to detect stage $V$ gametocytes circulating in the host blood and Pfs25-NASBA was performed as previously described [10, 40, 41]. The Pfs 25 NASBA assay was carried out in a volume of $10 \mu \mathrm{L}$ per reaction and $2.5 \mu \mathrm{L}$ of isolated RNA was used in each reaction. Positivity was calculated with the time-to-positivity at the fluorescence passed a given threshold, the mean fluorescence of three negative controls plus 20SD [10]. In order to measure the gametocyte density, a standard curve was made in duplicate by 10 -fold serial diluted 3D7 stage V gametocytes (from $1.8 \times 10^{4}-1.8 \times 10^{-2}$ gametocytes $/ \mu \mathrm{L}$ ) in culture blood (Johns Hopkins University, Malaria Research Institute, Baltimore MD). The limit of quantification (LOQ) for Pfs25-NASBA was 0.018 gametocytes $/ \mu \mathrm{L}$ based on the standard curve established in this laboratory.

\section{Gametocyte diversity assessed by Pfg377 RT-PCR and $R T-q P C R$}

For detection of gametocyte diversity, Pfg377 reverse transcription polymerase chain reaction (RT-PCR) was performed according to a published protocol [42]. The Pfg377 mRNA are only expressed in female gametocytes from stage III onward [43, 44] and the Pfg377 gene contains four regions of repetitive sequence, the most polymorphic being region 3 . This assay was designed based on the most polymorphic region 3 , which encodes seven degenerate amino acid repeats ( 21 base pairs) for identification of multiple gametocyte clones within host [43]. Pfg377 RT-PCR was performed using SuperScript III onestep RT-PCR system with platinum Taq RNA polymerase
(Thermo Fisher Scientific, Waltham MA). Concurrently, a conventional DNA PCR was performed using the same RNA sample to rule out contamination with genomic DNA. RT-PCR products were run on a $4 \%$ UltraPure agarose 1000 gel (Thermo Fisher Scientific, Waltham MA) with 50-bp molecular weight standards. DNA band sizes were visualized and measured using the gel imaging system and Labworks image acquisition and analysis software v4.6 (UVP BioImaging Systems, Upland, CA). Multiple gametocyte alleles was assessed based on number of bands and differences in band size [45].

For quantification of Pfg377 mRNA, quantitative reverse transcription (RT-qPCR) of Pfg377 was performed as described previously [46]. The standard curve was made with the purified RNA from stage IV and V mature gametocytes by 10 -fold dilution series from $10^{4}$ to $10^{0}$ gametocytes per $\mu \mathrm{L}$ of blood. All specimens were tested in duplicate; the mean $\mathrm{Ct}$ and number of gametocytes were obtained automatically on the Stratagene Mx3005P qPCR system (Agilent Technologies Inc., Santa Clara, CA). The limit of quantification (LOQ) for Pfg377 RT-qPCR was one parasites $/ \mu \mathrm{L}$ based on the standard curve established in this laboratory.

\section{Data sources and statistical analysis \\ Data sources}

The 18S-NASBA was used for measuring total parasite load and Pfs25-NASBA was used for measuring gametocyte carriage in both Asembo and Karemo study areas. In addition, Pfg377 RT-PCR and RT-qPCR were performed only on the samples collected in Asembo area for exploring the gametocyte diversity due to funding constraints. Because NASBA uses different approaches for determination of positivity and quantification of parasite density (see NASBA method section above), the positive values for samples with extremely low parasite density could have been below the cut-off of quantitative standard curve, in such positive samples a density was assigned at the midpoint between the cut-off and zero.

\section{Data analysis}

All data management was performed in SAS 9.3 (SAS Institute Inc., Cary, NC). Population weighted prevalence of parasitaemia and gametocytaemia was calculated via PROC SURVEYFREQ, accounting for field and laboratory sampling scheme and within-household clustering via Taylor series linearization [47]. Model selection and analysis was performed via the MuMIn package [48] and survey packages [49] in R (Version 3.1.3, R Development Core Team, Vienna, Austria) via RStudio (Version 0.98.1073, RStudio Team, Boston, MA).

Multivariable analysis was performed to evaluate participant characteristics associated with parasite or 
gametocyte presence and density, as well as characteristics associated with presence of multiple gametocyte alleles. Model selection was based on the generalized linear model (GLM) with either Gaussian distribution for continuous outcomes or binomial distribution with a logit link as appropriate for binary outcomes. Best fitting models were selected by lowest Bayesian information criterion (BIC) [50], and full models were then run in the survey package [49] (svyglm and svycontrast) to obtain appropriate odds ratios and confidence intervals for clustered data. Means and confidence intervals (95\% CI) for parasite density were calculated based on the observed marginals.

Association between gametocyte presence and participant characteristics was restricted to individuals who were $18 \mathrm{~S}$ positive and, therefore, considered infected. Data on parasite and gametocyte density were $\log _{10}$ transformed prior to analysis, so estimates are presented as geometric means. Study area was included as a class variable in all models. Where there was no interaction or main effect of study area, overall estimates were presented. Where there was significant impact of study area, area-specific results were presented. Age category of participants was included in all models. The sample collection time was included as a class variable where lowest BIC models included week to account for trends across the sampling timeframe. Anaemia was evaluated as a potential predictor in models for gametocytaemia only. Interactions were not evaluated for gametocyte diversity due to small sample size.

\section{Results}

\section{Characteristics of study participants}

Three smear-positive samples from Karemo were excluded from analysis due to absence of information on fever in previous $24 \mathrm{~h}$, leaving 550 samples from Karemo in this analysis. Thus, characteristics of participants for the study described here were from 996 individuals with complete data. Among 996 participants, $19.8 \%$ of participants reported fever in the past $24 \mathrm{~h}$ while $67.5 \%$ of individuals reported sleeping under an ITN the night prior to the survey. Among the 996 participants, $17.8 \%$ had used anti-malarials within the 2 weeks prior to the survey, of which more than $96 \%$ used was AL. Using the haemoglobin cut-off of $<11.0 \mathrm{~g} / \mathrm{dl}, 40.3 \%$ of individuals were considered anaemic.

\section{Parasite and gametocyte profiles by $18 \mathrm{~S}$ - and Pfs25-NASBA} in Asembo and Karemo

Out of the 996 samples from Asembo and Karemo, 848 $(85.1 \%)$ were $18 \mathrm{~S}-\mathrm{NASBA}$ positive (Table 1). Among them, $69.6 \%(334 / 480)$ of smear-negative samples tested demonstrated submicroscopic infection when measured by $18 \mathrm{~S}-\mathrm{NASBA}$ (Table 1). In Asembo, 18S-NASBA detected 220 (99.6 \%) positives from 221 smear-positive samples and identified 135 (60.0\%) positives from 225 smear-negative samples tested (Table 1). In comparison, 18S-NASBA detected 294 (99.7\%) positives from 295 smear-positive samples and 199 (78.0 \%) positives from 255 smear-negative samples tested in Karemo (Table 1). Overall, weighted population prevalence of parasitaemia using smear diagnosis was $30.6 \%$ (CI 26.2-35.0\%), relative to a weighted prevalence of $80.2 \%$ (CI 79.2$84.2 \%$ ) by 18 S-NASBA (Fig. 1). Parasite density was low for many smear-negative samples tested, with $31.5 \%$ (267/848) NASBA positive samples below one parasites/ $\mu \mathrm{L}$ of blood. The distribution of parasite density among smear-positive and smear-negative individuals is shown in Additional file 1 , panel A.

Overall, 531 out of 996 (53.3\%) samples tested were gametocyte positive by Pfs25-NASBA (Table 1). Weighted population prevalence of gametocytaemia was estimated to be $2.6 \%$ (CI $1.3-3.9 \%$ ) by smear diagnosis, relative to $44.0 \%$ (CI 39.0-49.0 \%) by Pfs25-NASBA (Fig. 1). Distribution of gametocyte density by Pfs25NASBA in smear-positive samples had a wide range from $10^{-2}$ to $10^{3}$ gametocytes $/ \mu \mathrm{L}$, while maximum gametocyte density in smear-negative samples did not exceed $10^{2}$ gametocytes $/ \mu \mathrm{L}$ (Additional file 1, panel B).

\section{Gametocyte diversity assessed by Pfg377 RT-PCR in Asembo}

Pfg377 positivity was detected in 124 of 221 (56.1\%) smear-positive and in 6 of 225 (2.7\%) smear-negative individuals tested in Asembo. Six single gametocyte alleles were identified based on band size (range from 273-378 bps) (Fig. 2a). Out of the 130 Pfg377 positive samples, the single allele infections accounted for $76.2 \%$

Table 1 Positivity of Plasmodium falciparum parasitaemia and gametocytaemia among samples tested by study areas

\begin{tabular}{|c|c|c|c|c|c|}
\hline \multirow[t]{2}{*}{ Assay } & \multirow[t]{2}{*}{ Overall $(n=996)$} & \multicolumn{2}{|l|}{ Asembo } & \multicolumn{2}{|l|}{ Karemo } \\
\hline & & $\begin{array}{l}\text { Smear-positive } \\
(n=221)\end{array}$ & $\begin{array}{l}\text { Smear-negative } \\
(n=225)\end{array}$ & $\begin{array}{l}\text { Smear-positive } \\
(n=295)\end{array}$ & $\begin{array}{l}\text { Smear-negative } \\
(n=255)\end{array}$ \\
\hline 18S-NASBA, n (\%) & $848(85.1)$ & 220 (99.6) & $135(60)$ & $294(99.7)$ & $199(78.0)$ \\
\hline Pfs25-NASBA, n (\%) & $531(53.3)$ & $177(80.1)$ & $24(10.7)$ & $259(87.8)$ & $71(27.8)$ \\
\hline
\end{tabular}




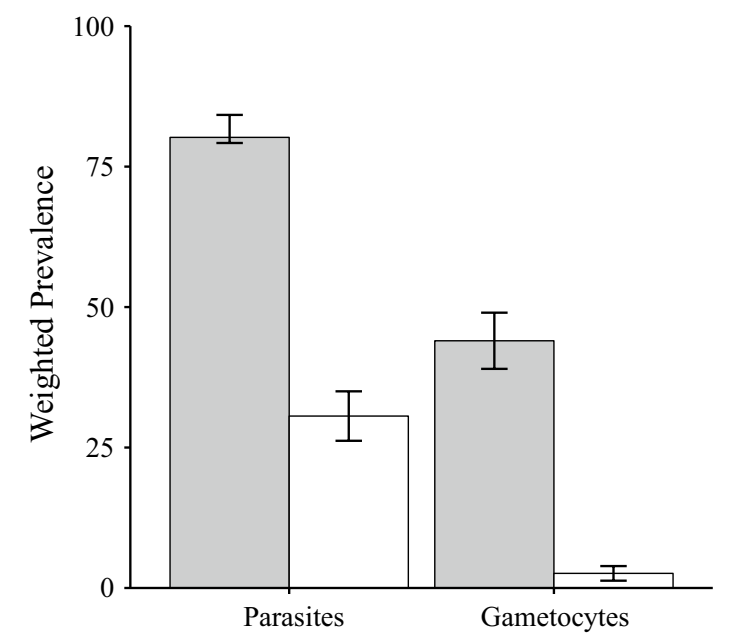

Fig. 1 Weighted prevalence of parasitaemia and gametocytaemia measured by 18S-NASBA and Pfs25-NASBA compared to blood smear method for residents of Asembo and Karemo, western Kenya, 2012. It shows weighted parasite prevalence of $80.2 \%$ by $185-N A S B A$ (grey filled bars) vs $30.6 \%$ by slide smear (white open bars) and weighted gametocyte prevalence of $44.0 \%$ by Pfs $25-N A S B A$ (grey filled bars) vs $2.6 \%$ by slide smear (white open bars) $(\mathrm{n}=99)$ and 31 samples were multiple alleles including two alleles $(\mathrm{n}=19)$, three alleles $(\mathrm{n}=10)$ and four alleles $(\mathrm{n}=2)$ within an infection (Fig. 2b). Both single band and multiple bands from the different combination of the six single alleles were seen on agarose gel (Fig. 2c, d).

\section{Risk factors for the presence of parasitaemia and gametocytaemia}

Adjusted odds ratios (aOR) for parasite or gametocyte presence and association with predictors were calculated based on the data of parasite and gametocyte presence in 996 individuals tested (Additional file 2). Overall, age influenced the presence of parasites and gametocytes. Older children (5-15 years old) were more likely to be parasitaemic than individuals $>15$ years old (OR 2.80, CI $1.46-5.38)$. Young children ( $<5$ years old) were less likely to be parasitaemic than older children (OR 0.52, CI 0.290.95 ), but not different compared to individuals $>15$ years old (OR 1.47, CI 0.93-2.31) (Table 2). Similarly, among $18 \mathrm{~S}$ positive individuals, school aged children were more likely to be gametocytaemic than individuals $>15$ years old (Pfs25: OR 3.37 CI 2.12-5.36), and young children
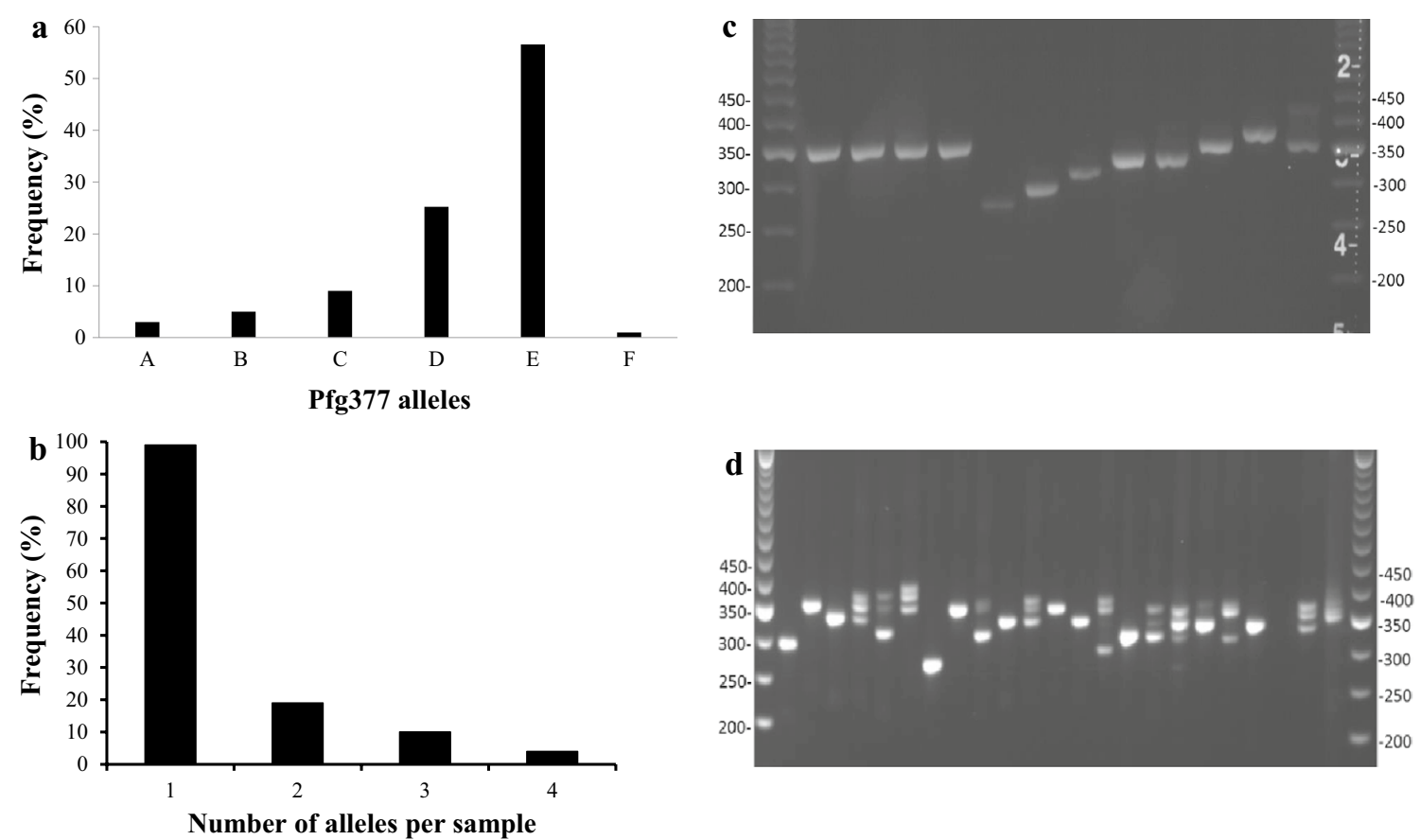

Fig. 2 Gametocyte diversity in human blood samples from Asembo, western Kenya $(n=130), 2012$. a shows the frequency of each single allele of gametocytes detected by Pfg377 RT-PCR. The predominant genotype (56.57 \%) is allele E (size 357 bp). b shows frequency distribution of Pfg 377 alleles per sample. c shows each single band on $4 \%$ electrophoresis gel. $\mathbf{d}$ shows the single or multiple bands of Pfg 377 region 3 . The gels were run with Tracklt ${ }^{\mathrm{TM}} 50$ bp DNA ladders (Thermo Fisher Scientific, MA) 
Table 2 Adjusted odds ratios (aOR) of parasite and gametocyte presence stratified by risk factor and intervention

\begin{tabular}{llll}
\hline aOR & Factor & Overall & Asembo \\
\hline 185 & Age: $<5$ vs $>15$ & $1.47(0.93-2.31)$ & \\
& Age: $5-15$ vs $>15$ & $2.80(1.46-5.38)^{\mathrm{a}}$ & \\
& Age $<5$ vs 5-15 & $0.52(0.29-0.95)^{\mathrm{a}}$ & \\
& Anaemia vs No Anaemia & NA & $0.26(0.10-0.68)^{\mathrm{a}}$ \\
& Fever vs No Fever & $1.21(0.75-1.95)$ & \\
& ITN vs No ITN & & \\
& AM vs No AM & $1.02(0.61-1.70)$ & \\
& Age: $<5$ vs $>15$ & $1.43(0.95-2.12)$ & \\
Pfs25 & Age: $5-15$ vs $>15$ & $3.37(2.12-5.36)^{\mathrm{a}}$ \\
& Age: $<5$ vs 5-15 & $0.42(0.28-0.64)^{\mathrm{a}}$ & \\
& Anaemia vs No Anaemia & $2.11(1.52-2.94)^{\mathrm{a}}$ & \\
& Fever vs No Fever & $1.65(1.11-2.46)^{\mathrm{a}}$ & \\
& ITN vs No ITN & $0.78(0.54-1.14)$ & \\
& AM vs No AM & $0.32(0.21-0.50)^{\mathrm{a}}$ & \\
\hline
\end{tabular}

Adjusted odds ratios accounting for multivariate comparisons ( $18 \mathrm{~S}$ included age, fever, ITN use, anti-malarial use, study area; Pfs 25 included age, fever, ITN use, anti-malarial use, study area, and anaemia). Age was stratified by set age categories $(<5,5-15,>15)$ in an adjusted analysis. Values were presented by overall for all parameters except for ITN vs No ITN for $18 \mathrm{~S}$ where there was an interaction by study area and risk factor, reporting by area

a Statistically significant

b Not analysed because parasitaemia causes anaemia

were less likely to be gametocytaemic than older children (OR 0.42 CI 0.28-0.64). Young children were not different compared to individuals $>15$ years old in odds for gametocytaemia (OR 1.43, CI 0.95-2.12) (Table 2).

Anaemia was associated with higher odds of gametocytaemia (OR 2.11, CI 1.52-2.94) (Table 2). Reported fever was also associated with higher odds of gametocytaemia (OR 1.65 CI 1.11-2.46) (Table 2). ITN use was associated with lower odds for parasitaemia in Asembo (OR 0.26, CI 0.1-0.68), but not in Karemo (OR 1.08, CI 0.60-1.93) (Table 2). Anti-malarial (AM) use within the past 2 weeks was associated with lower odds of gametocytaemia by Pfs25 (OR 0.32, CI 0.21-0.50) (Table 2). No other significant predictors were observed in the models predicting parasite or gametocyte presence.

\section{Risk factors for density of parasitaemia and gametocytaemia}

The density of parasitaemia and gametocytaemia decreased with age, with individuals $>15$ years old having the lowest density for both measures (18S and Pfs 25 all $\mathrm{p}<0.001$ ) (Fig. 3a). Parasite density did not differ between children under 5 and those $5-15$ years of age $(p=0.76)$, but gametocyte density was lower in older children relative to younger children $(\mathrm{p}=0.013)$. Anaemia was not associated with gametocyte density $(\mathrm{p}=0.77)$ (Fig. $3 \mathrm{~b})$. Geometric mean parasite density by $18 \mathrm{~S}-\mathrm{NASBA}$ was 7.9 times higher among individuals who had fever in the past $24 \mathrm{~h}(\mathrm{p}<0.001)$, but gametocyte density by Pfs 25 NASBA was not influenced by fever $(\mathrm{p}=0.68)$ (Fig. 3c).
There was no significant association between ITN use and parasite density $(\mathrm{p}=0.06)$ or gametocyte density $(\mathrm{p}=0.57)$ (Fig. 3d). Parasite density was lower among those who had received anti-malarials (AM) in the past 2 weeks $(p<0.001)$, while gametocyte density was not significantly different between individuals using $\mathrm{AM}$ and not using AM ( $\mathrm{p}=0.067$ ) (Fig. 3e).

\section{Risk factors for multiplicity of gametocyte infection in Asembo}

Among Pfg377 positive individuals, age, anaemia and recent anti-malarial treatment did not influence the probability of having multiple gametocyte alleles. However, the odds of having multiple gametocyte alleles were lower in individuals using ITNs than in those not using ITNs (OR 0.22, CI 0.07-0.68, $\mathrm{p}=0.0088$ ) (Fig. 4). High parasite density evaluated by $18 \mathrm{~S}-\mathrm{NASBA}$ (each 10 -fold increase relative to the mean of $4 \times 10^{2}$ parasites $/ \mu \mathrm{L}$ ) was associated with higher probability of having multiple gametocyte alleles (OR 2.78, CI 1.25-6.19, $\mathrm{p}=0.0126$ ) (Fig. 4), but high gametocyte density by Pfg377 was not associated with multiple gametocyte alleles $(\mathrm{p}=0.63)$.

\section{Discussion}

This study was conducted to estimate submicroscopic infection and gametocyte carriage of $P$. falciparum measured by sensitive RNA-based detection methods and to further assess the risk factors and interventions influencing gametocyte carriage in a community-based cross-sectional survey carried out in western Kenya in 

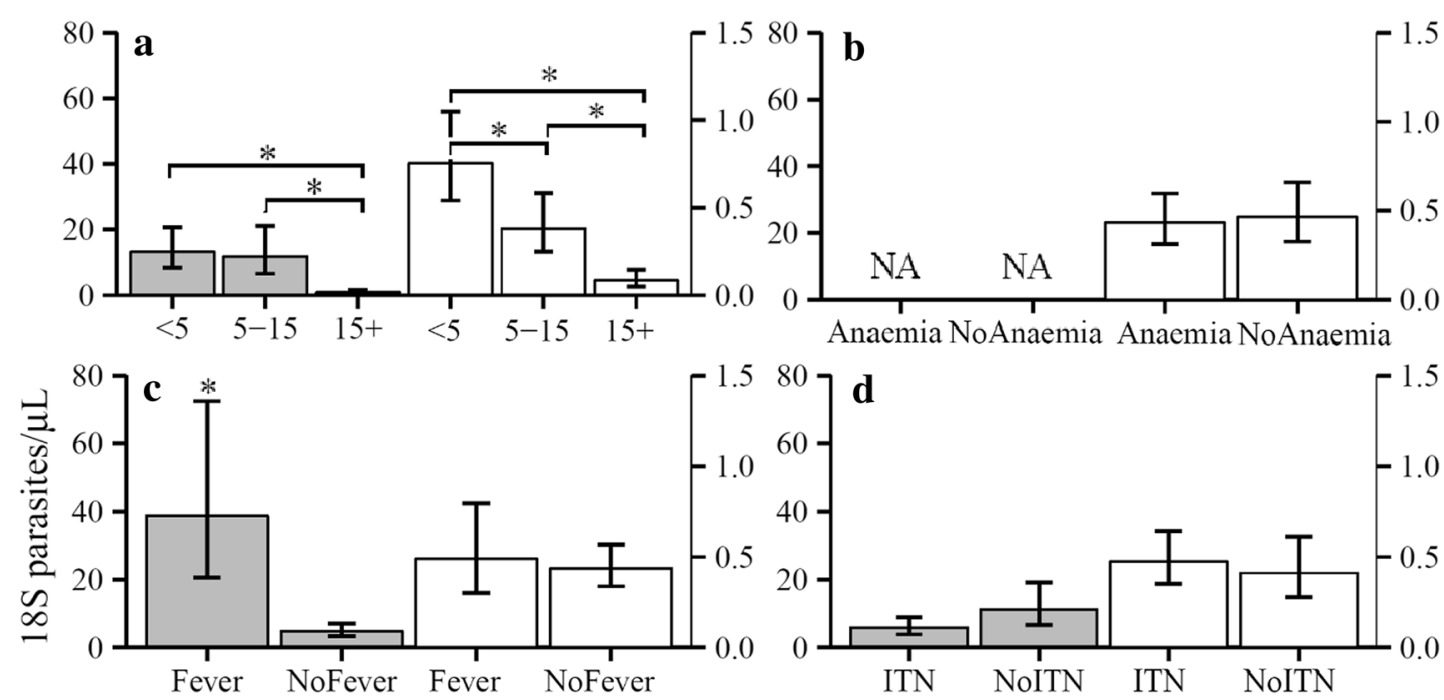

$807 \mathbf{d}$
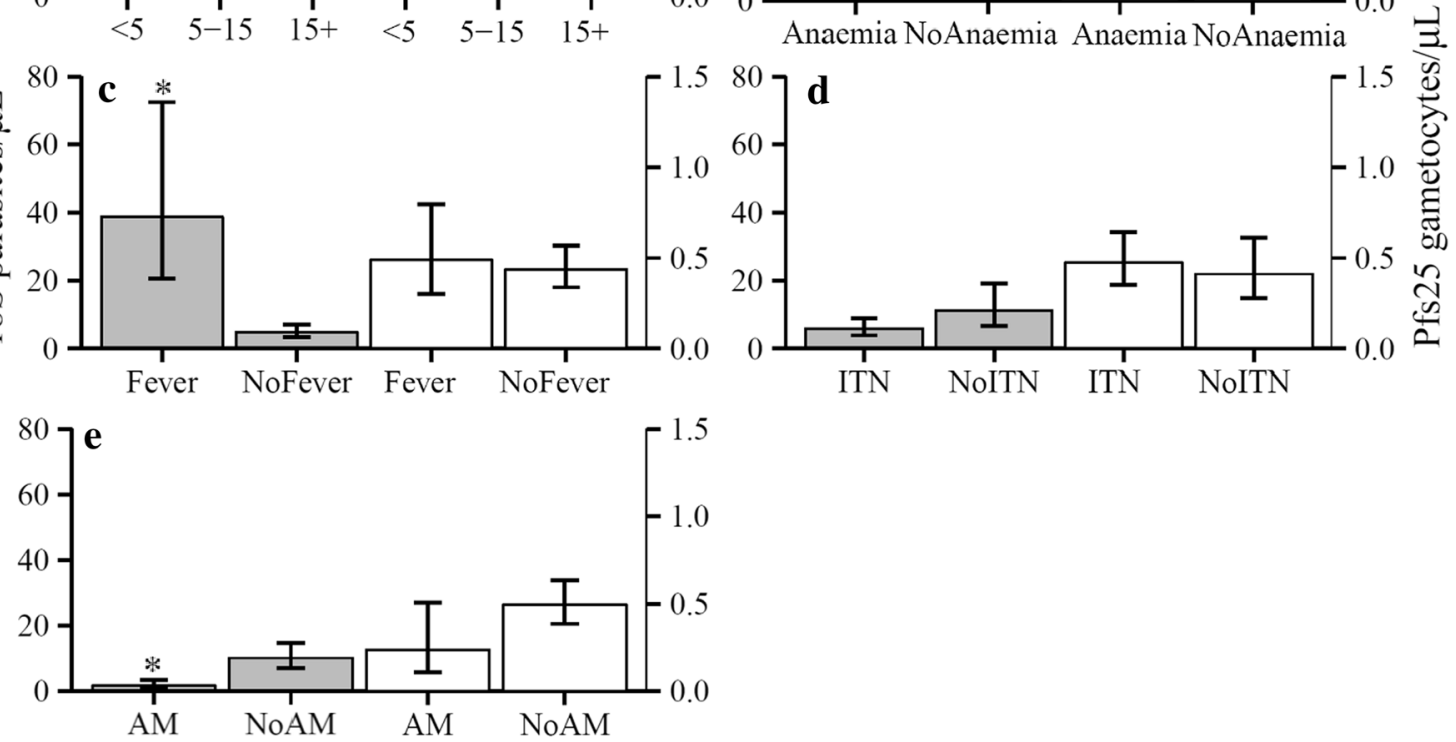

Fig. 3 Density of parasites and gametocytes and association with risk factors. Mean densities ( $\pm 95 \%$ Cl) of parasitaemia by $18 \mathrm{~S}$ (grey filled bars) or gametocytaemia by Pfs 25 (white open bars) were estimated by GLM, and presented using observed marginals. Left $Y$ axis is parasites/ $\mu \mathrm{L}$, while right $Y$ axis is gametocytes $/ \mu \mathrm{L}$. The brackets indicates the columns compared. Asterisks indicates that the column is significantly different ( $p<0.05$ ) than the second column with same colour for all panels. a Density of parasite and gametocyte was lower in individuals $>15$ years old than young or old children by $18 \mathrm{~S}$ and Pfs 25 ( $p<0.001$ for all comparisons). b Gametocytaemia density did not differ between anaemic and non-anaemic (NoAnaemia) individuals. c Parasite density was higher in individuals with fever. $\mathbf{d}$ There was no associations of ITN use on density of parasitaemia or gametocytaemia. e Parasite density was lower among individuals who had received anti-malarials (AM) in the past 2 weeks, but gametocyte density was not significantly different between receiving AM and not receiving AM (NoAM)

2012 during peak malaria transmission season. Using a subset of samples from the survey, this study revealed an overall high level of submicroscopic parasitaemia (69.6\% of randomly selected smear negative samples) and gametocytaemia ( $53.3 \%$ of all $18 \mathrm{~S}$ positive samples tested). When population level estimates were further calculated taking into account of multiple-layer sampling scheme, again a large proportion of infections were submicroscopic. Overall, $80.2 \%$ of the weighted population sample was parasite positive by $18 \mathrm{~S}$-NASBA vs $30.6 \%$ by smear diagnosis and $44.0 \%$ of the population was gametocyte positive by Pfs 25 -NASBA vs $2.6 \%$ by smear diagnosis. Risk factors for gametocyte carriage included being 5-15 years old, anaemia and self-reported fever. The study further showed that the use of anti-malarials within the past 2 weeks was associated with lower odds of gametocytaemia, but not gametocyte density, relative to untreated individuals. Use of ITNs the night before the survey was associated with lower odds of parasitaemia and lower odds of multiple allele gametocyte infection in Asembo.

The overall high level of submicroscopic infection and gametocyte carriage observed in this study was mainly attributed to using the sensitive 18S- and Pfs25-NASBA methods. NASBA is an isothermal nucleic acid amplification reaction that amplifies mRNA in a dsDNA background [51]. The risk of carry-over contamination in NASBA is minimized by the advantage of performing the entire assay in one step closed-tube-format [52]. NASBA is also unique for direct detection of abundant mRNA transcripts, which increases sensitivity compared to DNA-based detection methods [53, 54]. Moreover, gametocyte prevalence assessed by Pfs25-NASBA can be 3.3- to 8 -fold higher compared to those assessed by microscopy $[9,10,55]$. Results from the present study were consistent with these previous studies. Although NASBA is highly sensitive in detection of parasites and gametocytes, exact quantification of mRNA by NASBA 


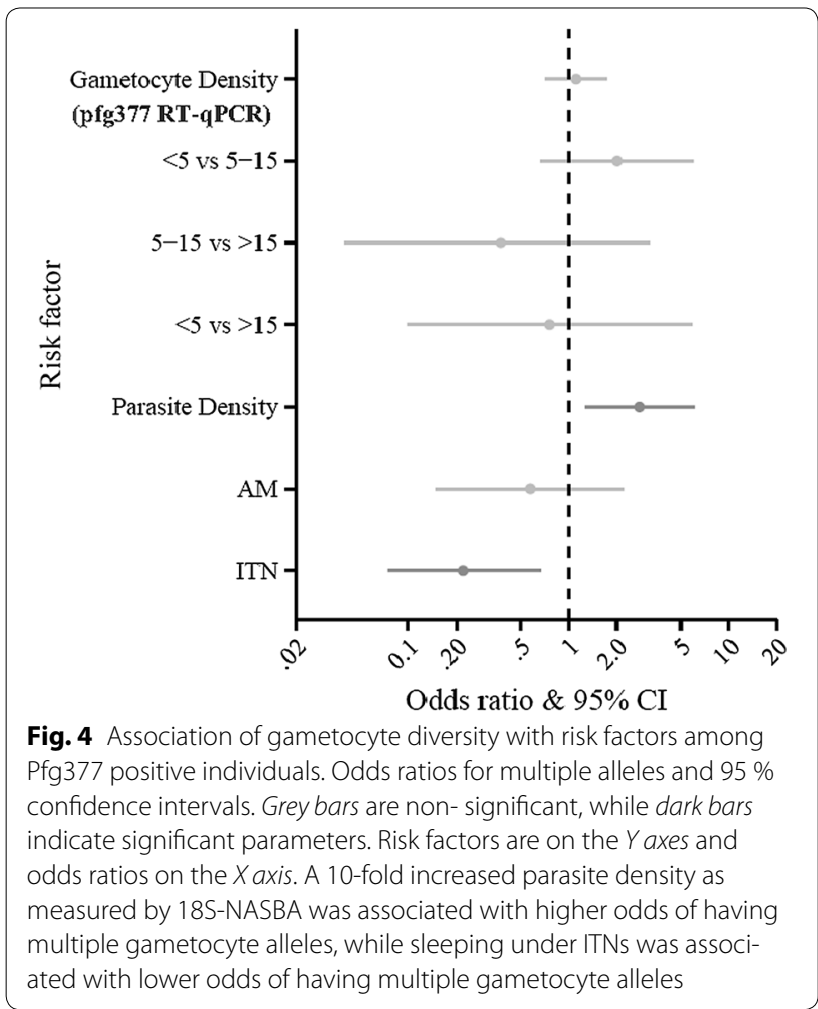

remains a challenge due to mRNA instability and loss during storage and extraction process, and inherent variability within NASBA amplifications. Therefore, the density measured by NASBA approach was served as a comparative estimate rather than a precise value.

Previous studies have suggested that a high prevalence of submicroscopic gametocytaemia may contribute to malaria transmission $[20,22,26,56]$. However, there is an argument that transmission is much less likely to occur at submicroscopic gametocyte levels [57]. The impact of extremely low gametocyte density detected by the sensitive Pfs25-NASBA on malaria transmission has not been established, although the value and usefulness of this method has been proven in estimating gametocyte prevalence in previous studies $[55,58]$ as well as in this cross-sectional survey. Moreover it is known that mere presence of gametocytes does not equate linearly to capacity to infect mosquitoes. And also, this study only measured mature gametocytes by Pfs 25 (infectious stage) in blood circulation, not including all immature gametocytes in circulation or sequestered in other tissues. Therefore, it was not a whole picture of gametocyte reservoir in host. In the present study, although there were many more NASBA gametocyte positives from smear-negative individuals (only a subset of smear-negative individuals were selected for this analysis), the majority of NASBA gametocyte positive individuals $(80 \%)$ were parasite smear-positive individuals. These results suggested that potential infectious reservoir could be predominantly contributed by individuals with smear-positive infections during peak transmission in the study areas. Together, these results showed a high level of submicroscopic infection and a large submicroscopic gametocyte stage $\mathrm{V}$ reservoir at community level, which may contribute to the stagnation in malaria prevalence in western Kenya.

Unlike a clear age-dependent decrease in densities of gametocytaemia (Fig. 3), older children (5-15 year old) had the highest odds for both parasitaemia and gametocytaemia (Table 2). The results from this study were not entirely consistent with previous reports of children $<5$ years old bearing the highest prevalence of asexual parasites and gametocytes in high endemic settings $[17,19,59-61]$. This new distribution pattern might be a result of the decreased EIR over 9-16 years of interventions in this area. However, the finding that children 5-15 years old were a significant reservoir for potential transmission was consistent with the recent malaria epidemiology in western Kenya [1, 2, 31]. Many factors might account for the unique age patterns for parasitaemia and gametocytaemia observed in the present study. Most likely, the highest odds of parasitaemia and gametocytaemia in older children was attributed to the older children not having been specifically targeted for malaria control programmes and the lower rates of ITN use among this age group $[2,31]$. On the other hand, the clear age-dependent decrease of density in gametocytes (Fig. 3a) most likely represented naturally acquired host immunity against both asexual and sexual stage parasites $[16,17,62,63]$. Whatever reasons for the variations between risk and density for parasitaemia and gametocytaemia, the results from this study indicated that children provided a larger gametocyte reservoir compared to adults in the peak transmission season in western Kenya assuming similar numbers of children and adults in population and similar mosquito bite rates received by children and adults.

Similar to previous studies [11, 64, 65], malaria-associated anaemia was related to the presence of gametocytes in this study. Anaemia is a common condition in $P$. falciparum infection due to the destruction of infected erythrocytes, shortened survival of uninfected erythrocytes and dyserythropoiesis [66-68]. It remains unclear to what extent which mechanisms are involved in the relationship between anaemia and gametocytogenesis. A high proportion of anaemic gametocytaemic individuals could be due to a longer duration of malarial infection $[69,70]$ or due to reticulocytes [71] and erythropoietin [72] triggering the pathway of gametocytogenesis. In addition, this study showed that high density of parasitaemia and presence of gametocytes measured by 
sensitive molecular tools were associated with fever. A previous study has reported that children $<15$ years old in Cameroon with asexual parasitaemia and gametocytes by microscopy have significantly higher prevalence of fever than those without gametocytes [73], while some other studies have reported that gametocyte presence detected by microscopy is negatively associated with fever in $P$. falciparum infection $[11,65]$. The relationship between gametocytes and fever is still incompletely understood [74]. But the association of fever with high density parasitaemia and presence of gametocytes observed in this study suggested that the individuals with fever might not get an effective malaria treatment at community level. Collectively, current results indicated that individuals with clinical malaria might be a major gametocyte reservoir in western Kenya.

$\mathrm{AL}$, a highly effective anti-malarial drug with gametocytocidal properties [75], was implemented in western Kenya as a first line treatment drug for uncomplicated malaria in 2006 [27]. In this study population, more than $96 \%$ of anti-malarial drugs reportedly used in the previous 2 weeks were AL. The results showed that, compared to individuals not receiving anti-malarial treatment, AL use within the past 2 weeks was associated with lower odds of gametocytaemia, but not of parasitaemia. In contrast, recent anti-malarial use was significantly associated with lowered parasite density, but not gametocyte density. The different responses to the drugs observed in this study confirmed that AL was more efficiently killing asexual parasites than mature gametocytes in infected individuals and then in turn decreased the chance of developing gametocytaemia in the infected persons. The current results were consistent with previous studies showing that ACT did not decrease proportion of individuals with malaria infection but was associated with a lower rate of gametocyte carriage $[26,29,76]$.

This study showed that sleeping under an ITN the night prior to the survey was associated with lower odds of parasitaemia by 18S-NASBA in Asembo, but not in Karemo and also did not influence gametocyte presence among parasitaemic individuals. The different effect of ITN use on the odds of parasitaemia between the two areas could possibly reflect relatively lower level of transmission in Asembo than in Karemo. Entomologic inoculation rates were relatively higher in Karemo in both 2011 and 2012 although the rates were below 10 infectious bites per person per year for both areas (Bayoh, unpublished data). However, these EIR measures were subject to high variation as they were a combined estimate of mosquito densities and sporozoite rates and easily affected by site selection and sampling methods. Parasite positivity as measured by microscopy and by 18S-NASBA in this study was similar between both areas suggesting the differences in EIRs were not substantial. Individual analysis and combined analysis were conducted by study area to explore if there were varying risk factors in these two adjacent areas since they had different ITN implementation time periods (7 years apart). Results from this study confirmed that there were no significant differences in risk factors between Asembo and Karemo except for ITN use on parasitaemia mentioned above.

This study showed that the higher parasite density by $18 \mathrm{~S}$ NSABA was associated with increased odds of gametocyte diversity. Previous studies have reported that high multiplicity of infection (MOI) is correlated with high malaria transmission level in endemic areas [77-79], and, in particular, is positively correlated with parasite density $[78,80]$. Higher parasite density might cause more frequent genetic recombination [80]. Also in this study, individuals reporting use of ITNs were less likely to have high gametocyte diversity. Interestingly, the result from the gametocyte Pfg377 mRNA measurement in this study differed from those of a previous study conducted in the same area. The previous study reported an increase of parasite diversity 5 years after ITN use [81] by assessing a Pfg377 DNA microsatellite locus within a coding region located upstream of region 3 of the Pfg377 gene [82]. It was speculated that the increase in Pfg377 microsatellite diversity at genomic level was a reflection of the parasite population adaptive and survival mechanisms due to reduced transmission [81]. The reason for the discrepancy in results between the gametocyte mRNA transcripts and genomic DNA remains unclear. A recent study in Burkina Faso has shown that only $60 \%$ of all Pfg377 positive samples contains at least one matching genotype between mRNA and genomic DNA [54]. It will be important to further investigate the relationship of gametocyte Pfg377 genetic diversities between stagespecific mRNA transcripts and genomic DNA in the context of malaria prevention and intervention.

This study had a few limitations. First, the sampling strategy of cross-sectional survey in 2012 involved randomly selecting a probability sample of compounds with at least one child under 5 years of age. Therefore, no weighing for geographical density of households was done. Second, self-reported ITN use, fever, recent AL treatment might introduce recall bias among study participants. Third, due to funding constraints, the total number of samples tested for 18S- and Pfs25-NASBA was limited, resulting in full coverage of smear positive samples, but a random selection of smear negative samples from the survey. Because of the multi-level stratified sampling scheme, complex analysis was employed for determination of population prevalence estimates. Both household and laboratory selections were addressed by weighted analysis and all weighted estimates were based on the subset of 
data selected for laboratory analysis. Although this was a subset of the entire epidemiological dataset with a complex study design, the statistical correction used in this study most likely resulted in representative population estimates of the study area. As well, for resource limited reason, Pfg377 diversity was tested in the samples from the one area with a longer history of ITN interventions for exploration. Although the gametocyte diversity was only examined in this area, the results clearly showed that use of ITNs had a protective role against the gametocyte diversity. The exploratory results could lead to further investigation of gametocyte diversity in relation to sex ratio in context of vector control in future.

\section{Conclusions}

This study showed a large proportion of submicroscopic parasites and gametocytes in western Kenya, which might partially explain stagnation in malaria prevalence and suggests that additional interventions are needed to target the infectious reservoir. As school aged children (5-15 years old) had the highest likelihood for both parasitaemia and gametocytaemia, this age group should be paid more attention in improving the ITN coverage in this area. Gametocyte presence was positively associated with fever and anaemia, indicating patients with clinical malaria or malaria-associated anaemia might be one of major sources for gametocyte reservoir and for potential malaria transmission in western Kenya. Recent AL use reduced parasite density and prevalence of gametocytes, but not the gametocyte density, indicating a limitation of AL in having an impact on transmission reservoir. ITN use played a protective role against parasitaemia and gametocyte diversity in an area with long-standing Asembo area of western Kenya.

\section{Additional files}

Additional file 1. Density distribution of parasites and gametocytes detected by 2 molecular assays. Histograms of parasite and gametocyte densities in age groups as quantified by molecular methods (18S-NASBA in Panel A and Pfs25-NASBA in Panel B). Histogram with filled grey bars represents smear-negative samples, while histogram with open bars represents smear-positive samples. $X$-axes represent the density of parasite (Panel A) or gametocytes (Panel B) per microliter of blood. Y-axes represents the number of positive individuals by molecular assays.

Additional file 2. Proportion of parasite and gametocyte presence in 996 samples tested by 185 -and Pfs25-NASBA stratified by risk factor and intervention. Proportions are reported in overall and each area, ${ }^{\$} \mathrm{NA}$ : not analysed because parasitaemia causes anaemia. surface antigen; Pfg377: 377 kDa female gametocyte-specific protein; LOQ: limit of quantification; GLM: generalized linear model; BIC: Bayesian information criterion; OR: odds ratio; $\mathrm{Cl}$ : confidence interval.

\section{Authors' contributions}

YPS, JG, EDW and ZZ conceived and designed the current study. ZZ performed the experiments. RMM and RW performed statistical analyses. MD, SK, $\mathrm{CO}, \mathrm{PO}, \mathrm{KO}, \mathrm{PO}$ and $\mathrm{VW}$ conducted or participated in the community-level cross-sectional survey in western Kenya including sample collection, haemoglobin and blood smear examinations, and data collection and management. ZZ, RMM and YPS wrote the manuscript. All authors read and approved the final manuscript.

\section{Author details}

${ }^{1}$ Malaria Branch, Division of Parasitic Diseases and Malaria, Center for Global Health, Centers for Disease Control and Prevention, Atlanta, GA, USA. ${ }^{2}$ Centre for Global Health Research, Kenya Medical Research Institute, Kisumu, Kenya. ${ }^{3}$ Department of Microbiology and Molecular Genetics, Michigan State University, East Lansing, MI, USA.

\section{Acknowledgements}

We thank Dr. Godfree Mlambo, Johns Hopkins Malaria Research Institute for providing 3D7 stage IV and V gametocytes and Dr. Naomi Lucchi, Malaria Branch, DPDM, CGH, CDC for providing 3D7 ring stage parasites as experiment controls. We thank HIV drug resistance team, International Laboratory Branch, DGHT, CGH, CDC for providing EasyQ Analyser. RMM is an ASM/CDC fellow sponsored by the fellowship programme of American Society of Microbiology. We are grateful to the communities of Rarieda and Karemo in Siaya County and caretakers who participated in the cross-sectional survey. We thank Dr. Patrick Kachur, the Chief of Malaria Branch, DPDM, CGH, CDC for his critical review on this manuscript and valuable suggestions. This paper was published with the permission of the director of the Kenya Medical Research Institute. The findings and conclusions in this paper are those of the authors and do not necessarily represent the official position of the Centers for Disease Control and Prevention.

\section{Competing interests}

The authors declare that they have no any competing interests.

\section{Availability of data and materials}

The datasets supporting the conclusions of this article can be found publicly within the main text and its additional files of this article.

\section{Consent for publication}

All authors read and approved the final manuscript.

\section{Ethics approval and consent to participate}

The protocol for this study was reviewed and approved by the Ethics Review Committee of the Kenya Medical Research Institute. The protocol was also reviewed and determined to be programme evaluation by the Centers for Disease Control and Prevention. Written informed consent was obtained from all participants or, in the case of children, from their parents or guardians.

\section{Funding}

This study was supported partially by U.S. National Science Foundation, Ecology of Infectious Diseases grant\# EF-0723770 and by Malaria Branch, Division of Parasitic Diseases and Malaria (DPDM), Center for Global Health (CGH), CDC with a cooperative agreement with the Kenya Medical Research Institute. The funders had no role in study design, data collection and analysis, decision to publish, or preparation of the manuscript.

Received: 14 June 2016 Accepted: 10 August 2016

Published online: 19 August 2016

\section{References}

1. Hamel MJ, Adazu K, Obor D, Sewe M, Vulule J, Williamson JM, et al. A reversal in reductions of child mortality in western Kenya, 2003-2009. Am JTrop Med Hyg. 2011;85:597-605.

\begin{abstract}
Abbreviations
ITN: insecticide-treated net; ACT: artemisinin-based combination therapy; AL: HDSS: Health and Demographic Surveillance System; DBS: dried blood spot; EIR: entomological inoculation rate; NASBA: nucleic acid sequence based amplification; 18S: 18S small subunit ribosomal RNA; Pfs25: 25 kDa ookinete
\end{abstract}


2. DOMC. Division of malaria control. 2010. Kenya malaria indicator survey http://www.malariasurveys.org/surveys.cfm?country=Kenya.

3. Aguilar R, Magallon-Tejada A, Achtman AH, Moraleda C, Joice R, Cistero $\mathrm{P}$, et al. Molecular evidence for the localization of Plasmodium falciparum immature gametocytes in bone marrow. Blood. 2014;123:959-66.

4. Bousema T, Drakeley C. Epidemiology and infectivity of Plasmodium falciparum and Plasmodium vivax gametocytes in relation to malaria control and elimination. Clin Microbiol Rev. 2011;24:377-410.

5. Eichner M, Diebner HH, Molineaux L, Collins WE, Jeffery GM, Dietz K. Genesis, sequestration and survival of Plasmodium falciparum gametocytes: parameter estimates from fitting a model to malariatherapy data. Trans $\mathrm{R}$ Soc Trop Med Hyg. 2001;95:497-501.

6. Bousema T, Okell L, Shekalaghe S, Griffin JT, Omar S, Sawa P, et al. Revisiting the circulation time of Plasmodium falciparum gametocytes: molecular detection methods to estimate the duration of gametocyte carriage and the effect of gametocytocidal drugs. Malar J. 2010;9:136.

7. Bousema T, Okell L, Felger I, Drakeley C. Asymptomatic malaria infections: detectability, transmissibility and public health relevance. Nat Rev Microbiol. 2014;12:833-40.

8. Churcher TS, Bousema T, Walker M, Drakeley C, Schneider P, Ouedraogo $\mathrm{AL}$, et al. Predicting mosquito infection from Plasmodium falciparum gametocyte density and estimating the reservoir of infection. Elife. 2013;2:e00626.

9. Schneider P, Wolters L, Schoone G, Schallig H, Sillekens P, Hermsen R, et al. Real-time nucleic acid sequence-based amplification is more convenient than real-time PCR for quantification of Plasmodium falciparum. J Clin Microbiol. 2005;43:402-5.

10. Schneider P, Bousema T, Omar S, Gouagna L, Sawa P, Schallig H, et al. (Sub)microscopic Plasmodium falciparum gametocytaemia in Kenyan children after treatment with sulphadoxine-pyrimethamine monotherapy or in combination with artesunate. Int J Parasitol. 2006;36:403-8.

11. von Seidlein L, Drakeley C, Greenwood B, Walraven G, Targett G. Risk factors for gametocyte carriage in Gambian children. Am J Trop Med Hyg. 2001;65:523-7.

12. Conway DJ, Roper C, Oduola AM, Arnot DE, Kremsner PG, Grobusch MP, et al. High recombination rate in natural populations of Plasmodium falciparum. Proc Natl Acad Sci USA. 1999;96:4506-11.

13. Daniels R, Chang HH, Sene PD, Park DC, Neafsey DE, Schaffner SF, et al. Genetic surveillance detects both clonal and epidemic transmission of malaria following enhanced intervention in Senegal. PLOS ONE. 2013;8:e60780.

14. Gbotosho GO, Sowunmi A, Happi CT, Okuboyejo TM. Plasmodium falciparum gametocyte carriage, sex ratios and asexual parasite rates in Nigerian children before and after a treatment protocol policy change instituting the use of artemisinin-based combination therapies. Mem Inst Oswaldo Cruz. 2011;106:685-90.

15. Makanga M. A review of the effects of artemether-lumefantrine on gametocyte carriage and disease transmission. Malar J. 2014;13:291.

16. Voller A, Cornille-Brogger R, Storey J, Molineaux L. A longitudinal study of Plasmodium falciparum malaria in the West African savannah using the ELISA technique. Bull World Health Organ. 1980:8:429-38.

17. Drakeley CJ, Akim NI, Sauerwein RW, Greenwood BM, Targett GA. Estimates of the infectious reservoir of Plasmodium falciparum malaria in The Gambia and in Tanzania. Trans R Soc Trop Med Hyg. 2000;94:472-6.

18. Shekalaghe SA, Bousema JT, Kunei KK, Lushino P, Masokoto A, Wolters $L R$, et al. Submicroscopic Plasmodium falciparum gametocyte carriage is common in an area of low and seasonal transmission in Tanzania. Trop Med Int Health. 2007;12:547-53.

19. Ouédraogo AL, Bousema T, de Vlas SJ, Cuzin-Ouattara N, Verhave J-P, Drakeley C, et al. The plasticity of Plasmodium falciparum gametocytaemia in relation to age in Burkina Faso. Malar J. 2010;9:281.

20. Schneider P, Bousema JT, Gouagna LC, Otieno S, van de Vegte-Bolmer M, Omar SA, et al. Submicroscopic Plasmodium falciparum gametocyte densities frequently result in mosquito infection. Am J Trop Med Hyg. 2007;6:470-4

21. Ouédraogo AL, Bousema T, Schneider P, de Vlas SJ, Ilboudo-Sanogo E, Cuzin-Ouattara N, et al. Substantial contribution of submicroscopical Plasmodium falciparum gametocyte carriage to the infectious reservoir in an area of seasonal transmission. PLoS ONE. 2009:4:e8410.

22. Karl S, Gurarie D, Zimmerman PA, King CH. St Pierre TG, Davis TM. A submicroscopic gametocyte reservoir can sustain malaria transmission. PLoS ONE. 2011;6:e20805
23. Griffin JT, Hollingsworth TD, Okell LC, Churcher TS, White M, Hinsley $W$, et al. Reducing Plasmodium falciparum malaria transmission in Africa: a model-based evaluation of intervention strategies. PLoS Med. 2010;7:e1000324.

24. Oguche S, Okafor HU, Watila I, Meremikwu M, Agomo P, Ogala W, et al. Efficacy of artemisinin-based combination treatments of uncomplicated falciparum malaria in under-five-year-old Nigerian children. Am J Trop Med Hyg. 2014;91:925-35.

25. Price RN, Nosten F, Luxemburger C, ter Kuile FO, Paiphun L, Chongsuphajaisiddhi $T$, et al. Effects of artemisinin derivatives on malaria transmissibility. Lancet. 1996;347:1654-8.

26. Bousema JT, Schneider P, Gouagna LC, Drakeley CJ, Tostmann A, Houben $R$, et al. Moderate effect of artemisinin-based combination therapy on transmission of Plasmodium falciparum. J Infect Dis. 2006;193:1151-9.

27. Amin AA, Zurovac D, Kangwana BB, Greenfield J, Otieno DN, Akhwale WS, et al. The challenges of changing national malaria drug policy to artemisinin-based combinations in Kenya. Malar J. 2007;6:72.

28. Beshir KB, Sutherland CJ, Sawa P, Drakeley CJ, Okell L, Mweresa CK, et al. Residual Plasmodium falciparum parasitemia in Kenyan children after artemisinin-combination therapy is associated with increased transmission to mosquitoes and parasite recurrence. J Infect Dis. 2013;208:2017-24.

29. Andagalu B, Mativo J, Kamau E, Ogutu B. Longitudinal study on Plasmodium falciparum gametocyte carriage following artemether-lumefantrine administration in a cohort of children aged 12-47 months living in Western Kenya, a high transmission area. Malar J. 2014;13:265.

30. Lengeler C. Insecticide-treated bed nets and curtains for preventing malaria. Cochrane Database Syst Rev. 2004;2:CD000363.

31. Desai M, Buff AM, Khagayi S, Byass P, Amek N, van Eijk A, et al. Agespecific malaria mortality rates in the KEMRI/CDC health and demographic surveillance system in western Kenya, 2003-2010. PLoS ONE. 2014;9:e106197.

32. Gimnig JE, Vulule JM, Lo TQ, Kamau L, Kolczak MS, Phillips-Howard PA et al. Impact of permethrin-treated bed nets on entomologic indices in an area of intense year-round malaria transmission. Am J Trop Med Hyg. 2003;68:16-22.

33. Nassir E, Abdel-Muhsin AM, Suliaman S, Kenyon F, Kheir A, Geha H, et al. Impact of genetic complexity on longevity and gametocytogenesis of Plasmodium falciparum during the dry and transmission-free season of eastern Sudan. Int J Parasitol. 2005;35:49-55.

34. Mitri C, Thiery I, Bourgouin C, Paul REL. Density-dependent impact of the human malaria parasite Plasmodium falciparum gametocyte sex ratio on mosquito infection rates. Proc Biol Sci. 2009;276:3721-6.

35. Burkot TR, Williams JL, Schneider I. Infectivity to mosquitoes of Plasmodium falciparum clones grown in vitro from the same isolate. Trans R Soc Trop Med Hyg. 1984;78:339-41.

36. Odhiambo FO, Laserson KF, Sewe M, Hamel MJ, Feikin DR, Adazu K, et al. The KEMRI/CDC health and demographic surveillance system - western Kenya. Int J Epidemiol. 2012;41:977-87.

37. Bayoh N, Walker ED, Kosgei J, Ombok M, Olang GB, Githeko AK, et al. Persistently high estimates of late night, indoor exposure to malaria vectors despite high coverage of insecticide treated nets. Parasit Vectors. 2014;7:380.

38. Wong J, Hamel MJ, Drakeley CJ, Kariuki S, Shi YP, Lal AA, et al. Serological markers for monitoring historical changes in malaria transmission intensity in a highly endemic region of Western Kenya, 1994-2009. Malar J. 2014;13:451.

39. Research aids. http://www.surveysystem.com/sscalc.htm. Accessed 10 Mar 2013.

40. Schneider P, Schoone G, Schallig H, Verhage D, Telgt D, Eling W, et al. Quantification of Plasmodium falciparum gametocytes in differential stages of development by quantitative nucleic acid sequence-based amplification. Mol Biochem Parasitol. 2004;137:35-41.

41. Schneider P, Reece SE, van Schaijk BC, Bousema T, Lanke KH, Meaden CS, et al. Quantification of female and male Plasmodium falciparum gametocytes by reverse transcriptase quantitative PCR. Mol Biochem Parasitol. 2015;199:29-33.

42. Menegon M, Severini C, Sannella A, Paglia MG, Sangare D, AbdelWahab A, et al. Genotyping of Plasmodium falciparum gametocytes by reverse transcriptase polymerase chain reaction. Mol Biochem Parasitol. 2000;111:153-61. 
43. Alano P, Read D, Bruce M, Aikawa M, Kaido T, Tegoshi T, et al. COS cell expression cloning of Pfg377, a Plasmodium falciparum gametocyte antigen associated with osmiophilic bodies. Mol Biochem Parasitol. 1995:74:143-56.

44. Alano P. Molecular approaches to monitor parasite genetic complexity in the transmission of Plasmodium falciparum malaria. Parassitologia. 2005:47:199-203.

45. Nwakanma D, Kheir A, Sowa M, Dunyo S, Jawara M, Pinder M, et al. High gametocyte complexity and mosquito infectivity of Plasmodium falciparum in the Gambia. Int J Parasitol. 2008;38:219-27.

46. Maeno Y, Nakazawa S, le Dao D, Yamamoto N, Giang ND, Van Hanh T, et al. A dried blood sample on filter paper is suitable for detecting Plasmodium falciparum gametocytes by reverse transcription polymerase chain reaction. Acta Trop. 2008;107:121-7.

47. Binder DA. On the variances of asymptotically normal estimators from complex surveys. Int Stat Rev. 1983;51:279-92.

48. Barton K. MuMIn: multi-model inference. R package version 1.15.6. 2016. https://cran.r-project.org/package=MuMln.

49. Lumley T. Analysis of complex survey samples. J Stat Softw. 2004;9:1-19.

50. Burnham KP, Anderson DR. Multimodel inference: understanding AIC and BIC in model selection. Sociol Methods Res. 2004;33:261-304.

51. Heim A, Grumbach IM, Zeuke S, Top B. Highly sensitive detection of gene expression of an intronless gene: amplification of mRNA, but not genomic DNA by nucleic acid sequence based amplification (NASBA). Nucleic Acids Res. 1998;26:2250-1.

52. Leone $G$, van Schijndel H, van Gemen B, Kramer FR, Schoen CD. Molecular beacon probes combined with amplification by NASBA enable homogeneous, real-time detection of RNA. Nucleic Acids Res. 1998;26:2150-5.

53. Schoone GJ, Oskam L, Kroon NC, Schallig HD, Omar SA. Detection and quantification of Plasmodium falciparum in blood samples using quantitative nucleic acid sequence-based amplification. J Clin Microbiol. 2000;38:4072-5.

54. Wampfler R, Timinao L, Beck HP, Soulama I, Tiono AB, Siba P, et al. Novel genotyping tools for investigating transmission dynamics of Plasmodium falciparum. J Infect Dis. 2014;210:1188-97.

55. Ouedraogo AL, Schneider P, de Kruijf M, Nebie I, Verhave JP, CuzinOuattara N, et al. Age-dependent distribution of Plasmodium falciparum gametocytes quantified by Pfs 25 real-time QT-NASBA in a cross-sectional study in Burkina Faso. Am J Trop Med Hyg. 2007;76:626-30.

56. Tietje K, Hawkins K, Clerk C, Ebels K, McGray S, Crudder C, et al. The essential role of infection-detection technologies for malaria elimination and eradication. Trends Parasitol. 2014:30:259-66.

57. Lin JT, Saunders DL, Meshnick SR. The role of submicroscopic parasitemia in malaria transmission: what is the evidence? Trends Parasitol. 2014;30:183-90

58. Marangi M, Di Tullio R, Mens PF, Martinelli D, Fazio V, Angarano G, et al. Prevalence of Plasmodium spp. in malaria asymptomatic African migrants assessed by nucleic acid sequence based amplification. Malar J. 2009;8:12.

59. Molineaux L, Storey J, Cohen JE, Thomas A. A longitudinal study of human malaria in the West African Savanna in the absence of control measures: relationships between different Plasmodium species, in particular P. falciparum and P. malariae. Am J Trop Med Hyg. 1980;29:725-37.

60. Akim NI, Drakeley C, Kingo T, Simon B, Senkoro K, Sauerwein RW. Dynamics of $P$. falciparum gametocytemia in symptomatic patients in an area of intense perennial transmission in Tanzania. Am J Trop Med Hyg. 2000:63:199-203.

61. Bousema JT, Gouagna LC, Drakeley CJ, Meutstege AM, Okech BA, Akim IN, et al. Plasmodium falciparum gametocyte carriage in asymptomatic children in western Kenya. Malar J. 2004;3:18.

62. Saeed M, Roeffen W, Alexander N, Drakeley CJ, Targett GA, Sutherland CJ. Plasmodium falciparum antigens on the surface of the gametocyteinfected erythrocyte. PLOS ONE. 2008;3:e2280.

63. Piper KP, Hayward RE, Cox MJ, Day KP. Malaria transmission and naturally acquired. Infect Immun. 1999;67:6369-74.
64. Price R, Nosten F, Simpson JA, Luxemburger C, Phaipun L, ter Kuile F, et al. Risk factors for gametocyte carriage in uncomplicated falciparum malaria. Am J Trop Med Hyg. 1999;60:1019-23.

65. Stepniewska K, Price RN, Sutherland CJ, Drakeley CJ, von Seidlein L, Nosten F, et al. Plasmodium falciparum gametocyte dynamics in areas of different malaria endemicity. Malar J. 2008;7:249.

66. Price RN, Simpson JA, Nosten F, Luxemburger C, Hkirjaroen L, ter Kuile $F$, et al. Factors contributing to anemia after uncomplicated falciparum malaria. Am J Trop Med Hyg. 2001;65:614-22.

67. White NJ, Ho M. The pathophysiology of malaria. Adv Parasitol. 1992;31:83-173.

68. Haldar K, Mohandas N. Malaria, erythrocytic infection, and anemia. Hematology Am Soc Hematol Educ Program. 2009;2009:87-93.

69. Metzger WG, Haywood M, D'Alessandro U, Drakeley CJ, Weiss H, Bojang $K$, et al. Serological responses of Gambian children to immunization with the malaria vaccine SPf66. Parasite Immunol. 1999:21:335-40.

70. Nacher M, Singhasivanon P, Silachamroon U, Treeprasertsuk S, Tosukhowong T, Vannaphan S, et al. Decreased hemoglobin concentrations, hyperparasitemia, and severe malaria are associated with increased Plasmodium falciparum gametocyte carriage. J Parasitol. 2002:88:97-101.

71. Trager W, Gill GS, Lawrence C, Nagel RL. Plasmodium falciparum: enhanced gametocyte formation in vitro in reticulocyte-rich blood. Exp Parasitol. 1999;91:115-8.

72. Reece SE, Duncan AB, West SA, Read AF. Host cell preference and variable transmission strategies in malaria parasites. Proc Biol Sci. 2005;272:511-7.

73. Sumbele IUN, Bopda OSM, Kimbi HK, Ning TR, Nkuo-Akenji T. Influence of Plasmodium gametocyte carriage on the prevalence of fever, splenomegaly and cardiovascular parameters in children less than 15 years in the Mount Cameroon area: cross sectional study. BMC Infect Dis. 2015; 15:547.

74. McKenzie FE, Jeffery GM, Collins WE. Gametocytaemia and fever in human malaria infections. J Parasitol. 2007;93:627-33.

75. Barnes Kl, Chanda P. Ab Barnabas G. Impact of the large-scale deployment of artemether/lumefantrine on the malaria disease burden in Africa: case studies of South Africa, Zambia and Ethiopia. Malar J. 2009;8(Suppl 1):S8.

76. Okell LC, Drakeley CJ, Ghani AC, Bousema T, Sutherland CJ. Reduction of transmission from malaria patients by artemisinin combination therapies: a pooled analysis of six randomized trials. Malar J. 2008;7:125.

77. Babiker HA, Ranford-Cartwright LC, Walliker D. Genetic structure and dynamics of Plasmodium falciparum infections in the Kilombero region of Tanzania. Trans R Soc Trop Med Hyg. 1999;93(Suppl 1):11-4.

78. Vafa M, Troye-Blomberg M, Anchang J, Garcia A, Migot-Nabias F. Multiplicity of Plasmodium falciparum infection in asymptomatic children in Senegal: relation to transmission, age and erythrocyte variants. Malar J. 2008;7:17.

79. Assefa SA, Preston MD, Campino S, Ocholla H, Sutherland CJ, Clark TG. estMOl: estimating multiplicity of infection using parasite deep sequencing data. Bioinformatics. 2014;30:1292-4.

80. Kateera F, Nsobya SL, Tukwasibwe S, Mens PF, Hakizimana E, Grobusch MP, et al. Malaria case clinical profiles and Plasmodium falciparum parasite genetic diversity: a cross sectional survey at two sites of different malaria transmission intensities in Rwanda. Malar J. 2016;15:237.

81. Gatei W, Kariuki S, Hawley W, ter Kuile F, Terlouw D, Phillips-Howard P, et al. Effects of transmission reduction by insecticide-treated bed nets (ITNs) on parasite genetics population structure: I. The genetic diversity of Plasmodium falciparum parasites by microsatellite markers in western Kenya. Malar J. 2010;9:353.

82. Anderson TJ, Haubold B, Williams JT, Estrada-Franco JG, Richardson L, Mollinedo R, et al. Microsatellite markers reveal a spectrum of population structures in the malaria parasite Plasmodium falciparum. Mol Biol Evol. 2000;17:1467-82. 